Boise State University

ScholarWorks

4-1-2012

\title{
Unusual Crystallite Growth and Modification of Ferromagnetism Due to Aging in Pure and Doped Zno Nanoparticles
}

Aaron Thurber

Boise State University

Gordon A. Alanko

Boise State University

Geoffrey L. Beausoleil II

Boise State University

Kelsey Dodge

Boise State University

Charles Hanna

Boise State University

See next page for additional authors 
Authors

Aaron Thurber, Gordon A. Alanko, Geoffrey L. Beausoleil II, Kelsey Dodge, Charles Hanna, and Alex Punnoose 


\title{
Unusual Crystallite Growth and Modification of Ferromagnetism Due to Aging in Pure and Doped Zno Nanoparticles
}

\author{
Aaron P. Thurber, Gordon Alanko, Geoffrey L. Beausoleil II, Kelsey N. Dodge, C. B. Hanna, and Alex \\ Punnoose \\ Boise State University
}

\begin{abstract}
We report the unusual growth of pure and $\mathrm{Fe}$ doped $\mathrm{ZnO}$ nanoparticles prepared by forced hydrolysis, and the weakening of ferromagnetism due to aging in ambient conditions. More than four dozen nanoparticle samples in the size range of $4-20 \mathrm{~nm}$ were studied over 1 to 4 years. The as-prepared samples had significant changes in their crystallite sizes and magnetization as they aged in ambient conditions. Detailed studies using x-ray diffraction and transmission electron microscopy (TEM) demonstrated that the crystallite size increased by as much as 1.4 times. Lattice parameters and strain also showed interesting changes. Magnetometry studies of $\mathrm{Zn}_{1-\mathrm{x}} \mathrm{Fe}_{\mathrm{x}} \mathrm{O}$ with $\mathrm{x}=0-0.2$ showed ferromagnetism at room temperature; however, keeping the samples in ambient conditions for one year resulted in modifications in the crystallite size and magnetization. For the $\mathrm{Zn}_{0.95} \mathrm{Fe}_{0.05} \mathrm{O}$ sample, the size changed from $7.9 \mathrm{~nm}$ to $9.0 \mathrm{~nm}$, while the magnetization decreased from $1 \mathrm{memu} / \mathrm{g}$ to 0.2 $\mathrm{memu} / \mathrm{g}$. Both magnetic and structural changes due to aging varied with the environment in which they were stored, indicating that these changes are related to the aging conditions.
\end{abstract}

\section{Introduction}

Metal oxide nanoparticles have received much attention and produced differing results regarding the existence of room temperature ferromagnetism, which has been observed in both pure and doped oxide nanoparticles of $\mathrm{SnO}_{2}$, $\mathrm{CeO}_{2}, \mathrm{ZnO}, \mathrm{TiO}_{2}$, and many other nanoparticles. ${ }^{1-11}$ The reported variations in the results and conclusions are quite controversial; however, it seems the magnetism observed in oxide nanoparticles may be affected by particle size, grain boundary area, defects, surfactant coating and/or doping. ${ }^{9,10,12-15}$ Upon revisiting samples which had been previously prepared, we noted that the estimated crystallite size had increased after several years. Prompted by our previous observation of an inverse relationship between particle size and magnetization ${ }^{15}$, we re-measured the magnetic properties of a number of aged samples and found many variable results. We believe that the apparent instability of these nanoparticles over time could be a key complicating factor leading to the current disagreements in experimental results. Our finding was unanticipated but potentially very important, so we undertook a detailed study to systematically investigate the effect of aging on the structural and magnetic properties of a large number of samples. We prepared pure and $\mathrm{Fe}$ doped $\mathrm{ZnO}$ nanoparticle samples and monitored their magnetic and structural properties as a function of age, in order to understand this unexpected aging behavior.

\section{Experimental Details}

Pure $\mathrm{Fe}$ doped $\mathrm{ZnO}$ nanoparticles at 1 and $5 \% \mathrm{Fe}$ were prepared by co-precipitation of appropriate amounts of $\mathrm{Zn}\left(\mathrm{OOCCH}_{3}\right)_{2} \cdot 2 \mathrm{H}_{2} \mathrm{O}$ and $\mathrm{Fe}\left(\mathrm{OOCCH}_{3}\right)_{2}$ in diethylene glycol by reflux heating of the reaction solution to $160{ }^{\circ} \mathrm{C}$. $0.2 \mathrm{~mL}$ of nanopure water was added to force hydrolysis. The sample was then extracted by centrifugation at 41,430 $\mathrm{g}$, washed in water and denatured ethanol, and finally dried at $50{ }^{\circ} \mathrm{C}$. Some samples were additionally coated with varying amounts of polyacrylic acid (PAA) by re-suspending the sample in $1 \mathrm{mM} \mathrm{NaCl}$ solution with either 0.25 or $10 \mathrm{mg} / \mathrm{mL}$ PAA. Some samples were prepared by a second similar method, in which the precursors were dissolved in $100 \mathrm{~mL}$ of $50 \mathrm{~g} / \mathrm{L} \mathrm{LiOH} \cdot \mathrm{H}_{2} \mathrm{O}$ in denatured ethanol, heated to $70{ }^{\circ} \mathrm{C}$ for 90 minutes, cooled, and mixed with 100 $\mathrm{mL}$ of $\mathrm{n}$-Heptane before centrifuging and washing. Samples were characterized shortly after preparation, before being stored in capped glass vials under ambient conditions. We re-characterized many of our samples when we first noticed unusual aging effects. Because these effects were unexpected, we performed these characterizations at varying lengths of time from initial sample preparation rather than in a set periodicity. X-ray diffraction (XRD) was performed using a Philips X'Pert MPD with $\mathrm{Cu} \mathrm{K}_{\alpha}$ radiation. Samples were mounted on a fixed horizontal plate in Bragg-Brentano geometry. Transmission electron microscopy (TEM) images were recorded on a JEOL JEM- 
Copyright 2012 American Institute of Physics. This article may be downloaded for personal use only. Any other use requires prior permission of

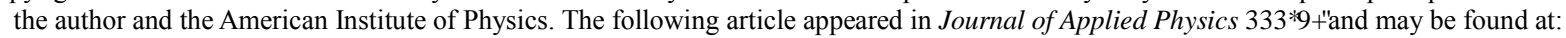
' 2 , $10.1063 / 1.3679147$.

2100HR microscope. Samples were suspended in denatured ethanol then dropped onto a carbon coated copper grid. Room temperature magnetic measurements were performed on a LakeShore 7404 vibrating sample magnetometer, with samples packed and sealed in a clear plastic straw.

\section{Results and Discussion}

Typical XRD data, as shown in Fig. 1a, always displayed only the pure wurtzite phase of ZnO. Peak width was used to determine crystallite size via the Scherrer relation. The sample shown here has a slight change in peak width (Fig. 1a, inset) over a 4 year period, demonstrating an increase in crystallite size from 5.1 to $7.9 \mathrm{~nm}$. The inset to Fig. 1a demonstrates the decrease in peak width of the aged sample more clearly. To confirm the change in size of the sample in Fig. 1a, TEM images from the as-synthesized sample and the same sample after 4 years of aging were collected and analyzed (Fig. 1b). The particle-size distribution (Fig. 1c) demonstrates a clear change in average particle size from 6.1 to $11 \mathrm{~nm}$. Furthermore, the particle size distribution is shown to broaden considerably. In order to see how the size variation depends on the initial sample size and Fe doping, a fresh set of nanoparticles with varying starting sizes and either 0 or $5 \%$ Fe was prepared;the results are shown in Fig. 2a. After 8 months, the rate of increase in size seems to be higher for larger starting samples, while the smallest sample sizes did not appear to change much over this shorter period. Doping with iron had no apparent impact. A normalized scatter plot of many $\mathrm{ZnO}$ nanoparticle samples re-characterized at varying ages (Fig. 2a, inset) demonstrates a general growth trend of the materials studied. The absolute amount of growth was nevertheless somewhat unpredictable.

One could potentially consider particles aged at room temperature to have been "sintered" over long time scales at $295 \mathrm{~K}$. If this were the case, kinetic grain growth should generally follow the rate equation

$$
\mathrm{G}^{\mathrm{n}}-\mathrm{G}_{0}^{\mathrm{n}}=\mathrm{k}_{0} \mathrm{t} \mathrm{e}^{(-\mathrm{Q} / \mathrm{RT})}
$$

with $\mathrm{n}$ between 2 and 4 . Ostwald ripening is a specific case with $\mathrm{n}=3$ for particles in colloidal suspension ${ }^{16,17}$ that is commonly seen in nanomaterials synthesis. Our powder samples do not seem to directly follow this equation for reasonable values of $\mathrm{n}$. Interestingly, a very recent report by Ali and Winterer proposed $\mathrm{Zn}$ interstitial diffusion promoted by adsorbed water as a mechanism for $\mathrm{ZnO}$ nanoparticle growth in powder form over a period of months ${ }^{18}$. In this model, long-term particle growth is strongly affected by the partial pressure of water, which may indicate a route to mitigation via very dry storage.

$\mathrm{M}$ vs. $\mathrm{H}$ data (Fig. 2b), shown for a 5\% Fe doped $\mathrm{ZnO}$ sample taken after synthesis and after 1 year of aging, shows a drastic decrease in magnetization, from $1 \mathrm{memu} / \mathrm{g}$ to $0.2 \mathrm{memu} / \mathrm{g}$. The same sample also displayed an increase in crystallite size as estimated by XRD, from 7.9 to $9.0 \mathrm{~nm}$. Such a variation is consistent with a previous observation that the magnetization decreases with increasing crystallite size in Fe doped $\mathrm{ZnO} .{ }^{15}$ In contrast, the same sample prepared with a $10 \mathrm{mg} / \mathrm{mL}$ PAA surface coating showed only a small decrease in magnetization (Fig. 2b), indicating that the surface PAA layer protects the magnetic properties of the nanoparticle. Although a decrease in saturation magnetization was a general trend in the behavior of aged particles, in the majority of samples studied (see Figs. $2 \mathrm{~b}$, $2 c, 3 a$ and $3 b$ ), there were several exceptions in which the magnetization increased after aging over the same period of time (Figure $3 \mathrm{~b}$ and $3 \mathrm{c}$ ). For example, similar nanoparticles coated with $0.25 \mathrm{mg} / \mathrm{mL}$ PAA resulted in an increase in magnetization after one year (Fig. 3b). Fig. 3a, which was prepared similarly to the sample shown in Fig. 2c, shows a very large drop in magnetization between the initial measurement and the measurement after aging for one year, while its 0.25 and $10 \mathrm{mg} / \mathrm{mL}$ coated counterparts displayed relatively little change.

In contrast to the behavior described above, while the sample in Fig. 3b dropped in magnetization when uncoated and aged for one year, its 0.25 and $10 \mathrm{mg} / \mathrm{mL}$ PAA coated counterparts showed large increases in magnetization. One $5 \%$ Fe doped $\mathrm{ZnO}$, starting at $5.7 \mathrm{~nm}$ when prepared, showed a large increase in magnetization after aging, while the sample coated with PAA showed no changes over time. An additional 5\% Fe doped $\mathrm{ZnO}(\sim 18 \mathrm{~nm}$ in size) had different saturation magnetizations afterward, whether uncoated, coated with 0.25 , or coated with $10 \mathrm{mg} / \mathrm{mL}$ PAA (Fig. 3c). These results may indicate that at least some surfactants may help stabilize particle size and magnetism by acting as a barrier to diffusion. Some of the uncertainties observed on the effect of aging on the magnetic properties of the surfactant coated nanoparticles might be related to the role played by the diamagnetic PAA surfactant. Aging over longer periods of time might have changed the amount of surfactant on the particles and caused cracks and imperfections on the surface layer. Since the primary goal of this work was to monitor the change 
Copyright 2012 American Institute of Physics. This article may be downloaded for personal use only. Any other use requires prior permission of

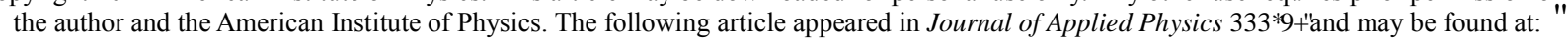

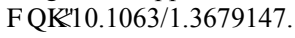

in the magnetization of each sample as a function of time, we did not measure the coating thickness in each nanoparticle system at any time point.

\section{Conclusions}

We have shown that the size and magnetic properties of $\mathrm{ZnO}$ nanopowders are unstable over month or year time scales when aging in ambient conditions, which may call into question previous measurements of the magnetic properties of $\mathrm{ZnO}$ nanomaterials, and explain the divergence in reported measurements in the literature. Changes in crystallite size are certain to be accompanied by changes in available grain boundary and/or interfacial area, as well as the concentration and types of defects, all of which have been theorized ${ }^{9,10,12}$ to be relevant to the observed magnetism in this material. Researchers should expect room-temperature, ambient-condition aging of $\mathrm{ZnO}$ nanoparticles to result in significant changes in magnetic properties. Storage under vacuum or in a d esiccating environment such as $\mathrm{CaCl}_{2}$ or silica gel may mitigate these effects. While more studies are necessary to monitor nanoparticle samples in different environments, it is already clear that sample synthesis procedures, sample history, and sample aging can heavily impact the observed magnetism.

\section{Acknowledgments:}

This work was supported in part by NSF EAGER DMR-1137419, ARO W911NF-09-1-0051 and NSF CBET 1134468 grants. C.B.H. was supported by a NSF DMR-0906618 grant. 


\section{References}

1. J. M. D. Coey, P. Stamenov, R. D. Gunning, M. Venkatesan, and K. Paul, New J. Phys. 12, 053025 (2010).

2. J. M. D. Coey, K. Wongsaprom, J. Alaria, and M. Venkatesan, J. Phys. D-Appl. Phys. 41, 134012 (2008).

3. T. Dietl, Nat. Mater. 9, 965965 (2010).

4. T. Dietl, H. Ohno, F. Matsukura, J. Cibert, and D. Ferrand, Science 287, 10191019 (2000).

5. J. Hays, A. Thurber, K. M. Reddy, A. Punnoose, and M. H. Engelhard, Journal of Applied Physics 99, 08M123 (2006).

6. J. Philip, A. Punnoose, B. I. Kim, K. M. Reddy, S. Layne, J. O. Holmes, B. Satpati, P. R. Leclair, T. S. Santos, and J. S. Moodera, Nat. Mater. 5, 298298 (2006).

7. A. Punnoose, J. Hays, A. Thurber, M. H. Engelhard, R. K. Kukkadapu, C. Wang, V. Shutthanandan, and S. Thevuthasan, Phys. Rev. B 72, 054402 (2005).

8. A. Punnoose, M. S. Seehra, W. K. Park, and J. S. Moodera, Journal of Applied Physics 93, 78677867 (2003).

9. B. Straumal, A. Mazilkin, S. Protasova, A. Myatiev, P. Straumal, E. Goering, and B. Baretzky, Phys. Status Solidi B-Basic Solid State Phys. 248, 15811581 (2011).

10. B. B. Straumal, A. A. Myatiev, P. B. Straumal, A. A. Mazilkin, S. G. Protasova, E. Goering, and B. Baretzky, Jetp Lett. 92, 396396 (2010).

11. A. Thurber, K. M. Reddy, V. Shutthanandan, M. H. Engelhard, C. Wang, J. Hays, and A. Punnoose, Phys. Rev. B 76, 165206 (2007).

12. D. Q. Gao, J. Zhang, J. Y. Zhu, J. Qi, Z. H. Zhang, W. B. Sui, H. G. Shi, and D. S. Xue, Nanoscale Res. Lett. 5, 769769 (2010).

13. X. Zuo, S.-D. Yoon, A. Yang, W.-H. Duan, C. Vittoria, and V. G. Harris, Journal of Applied Physics 105, 07C508 (2009).

14. J. F. Liu, E. Z. Liu, H. Wang, N. H. Su, J. Qi, and J. Z. Jiang, Nanotechnology 20, 165702 (2009).

15. A. P. Thurber, G. L. Beausoleil, G. A. Alanko, J. J. Anghel, M. S. Jones, L. M. Johnson, J. H. Zhang, C. B. Hanna, D. A. Tenne, and A. Punnoose, Journal of Applied Physics 109, 07C305 (2011).

16. G. Oskam, Z. Hu, R. L. Penn, N. Pesika, and P. C. Searson, Physical Review E 66, 011403 (2002).

17. R. Viswanatha, P. K. Santra, C. Dasgupta, and D. D. Sarma, Physical Review Letters 98, 255501 (2007).

18. M. Ali and M. Winterer, Chemistry of Materials 22, 85 (2010). 


\section{FIGURE CAPTIONS}

Figure 1. (a) XRD data of a pure $\mathrm{ZnO}$ sample before and after 4 years of aging. The inset is an expanded view to show the reduction in the width of one of the peaks more clearly. (b) TEM images of the same sample before and after aging. (c) particle-size distribution of the asprepared and aged samples calculated from the TEM images.

Figure 2. (a) XRD-estimated crystallite size of pure (filled squares) and 5\% Fe doped (open circles) $\mathrm{ZnO}$ nanoparticles from x-ray diffraction data aged over 8 months. The inset shows normalized growth vs. time for pure $\mathrm{ZnO}$ (filled black symbols) and 5\% Fe doped (open red symbols), for different initial size in the range 5-10 $\mathrm{nm}$ (circles) and 10-20 $\mathrm{nm}$ (squares). Also shown are $\mathrm{M}$ vs $\mathrm{H}$ data on (b) 5\%, and (c) $1 \%$ Fe doped $\mathrm{ZnO}$ samples, before (filled symbols) and after (hollow symbols) 1 year of aging, without (squares) and with $10 \mathrm{mg} / \mathrm{mL}$ PAA coating (circles).

Figure 3. $\mathrm{M}$ vs $\mathrm{H}$ data on (a) $1 \%$ Fe doped $\mathrm{ZnO}$ prepared in the smallest size $(\sim 6 \mathrm{~nm})$, (b) $1 \%$ Fe doped $\mathrm{ZnO}$ prepared in the largest size $(\sim 18 \mathrm{~nm})$, and (c) $5 \%$ Fe doped $\mathrm{ZnO}$ prepared in the largest size $(\sim 18 \mathrm{~nm})$. Filled symbols indicate as-prepared data, while empty symbols indicate data collected after approximately 1 year of aging. Squares represent samples without PAA coating, while circles represent samples with PAA coating at the concentration specified. 

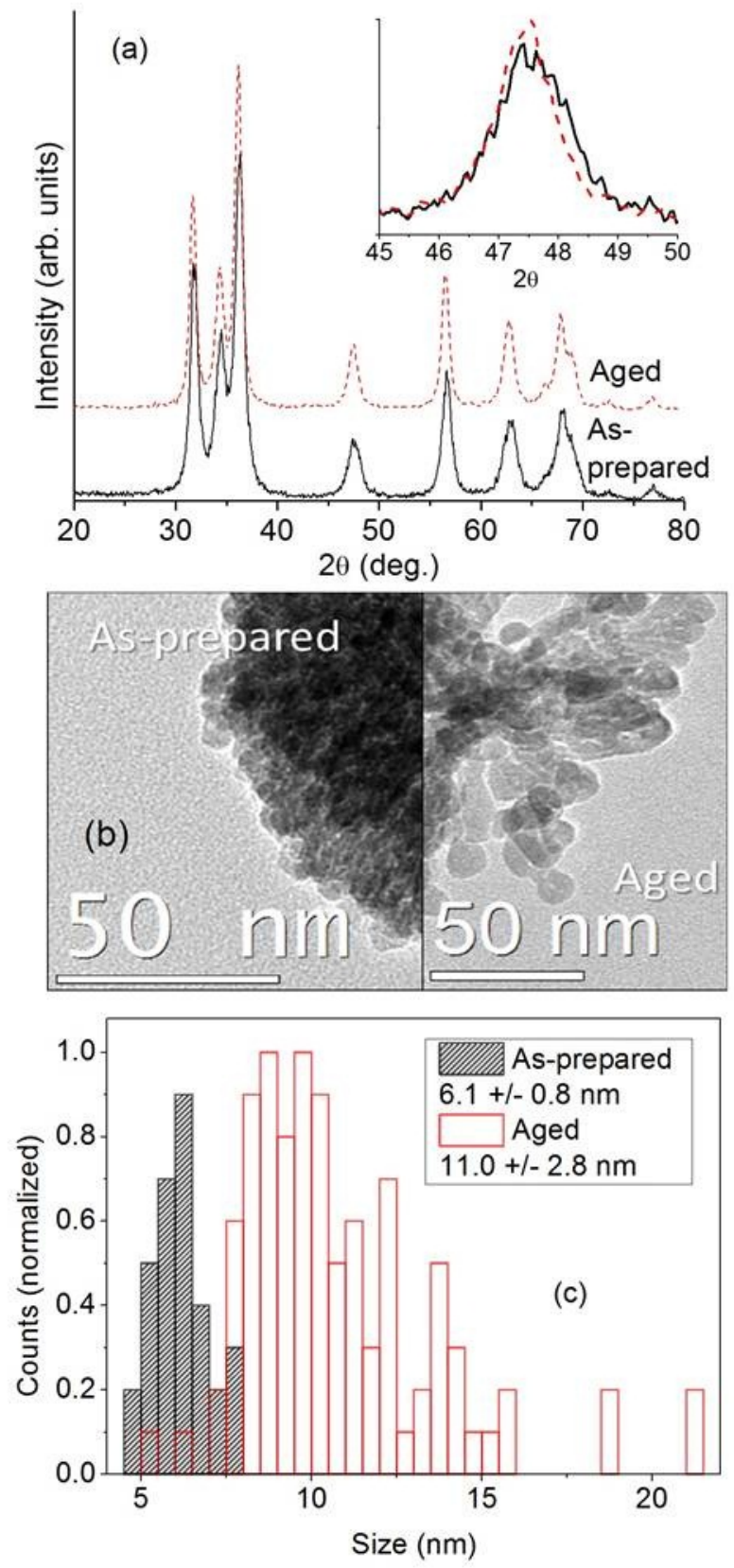
- Pure $\mathrm{ZnO} \circ 5 \% \mathrm{Fe}$
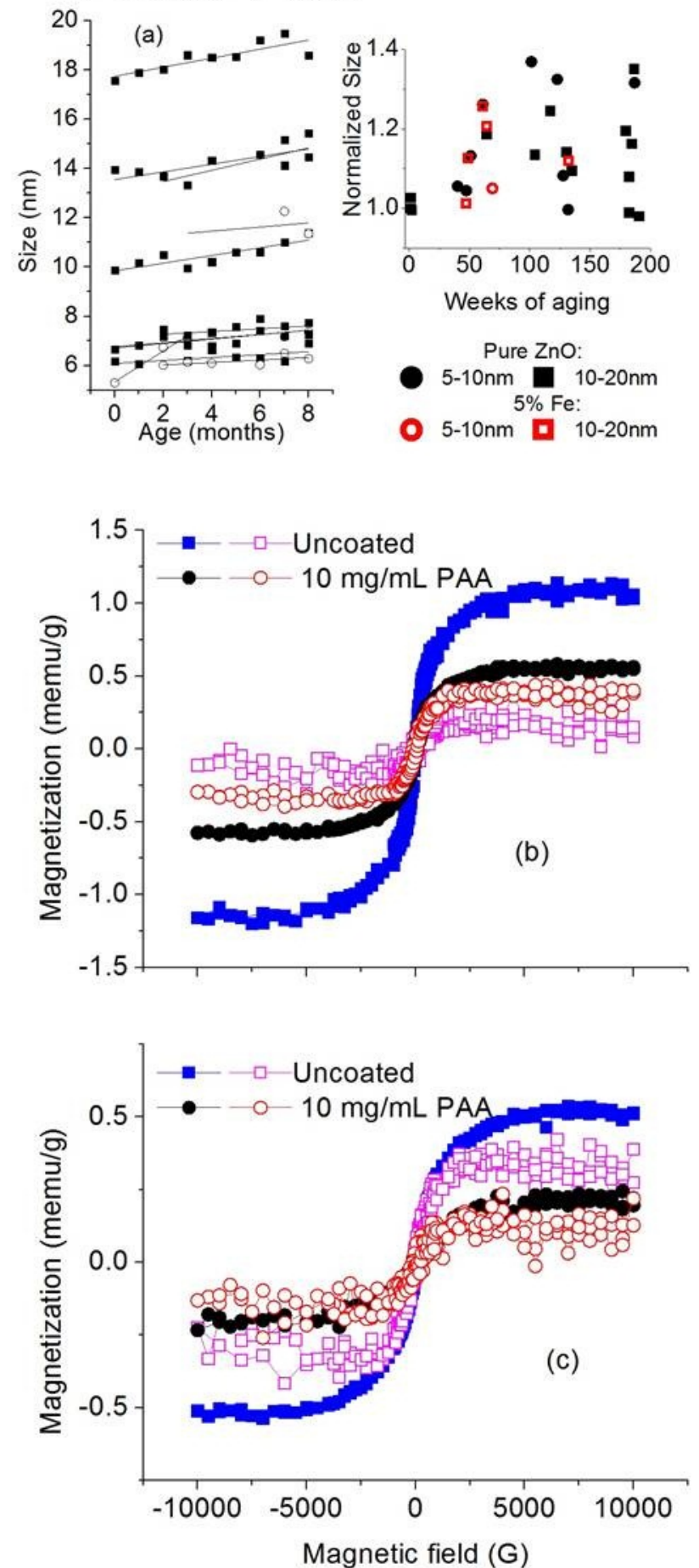


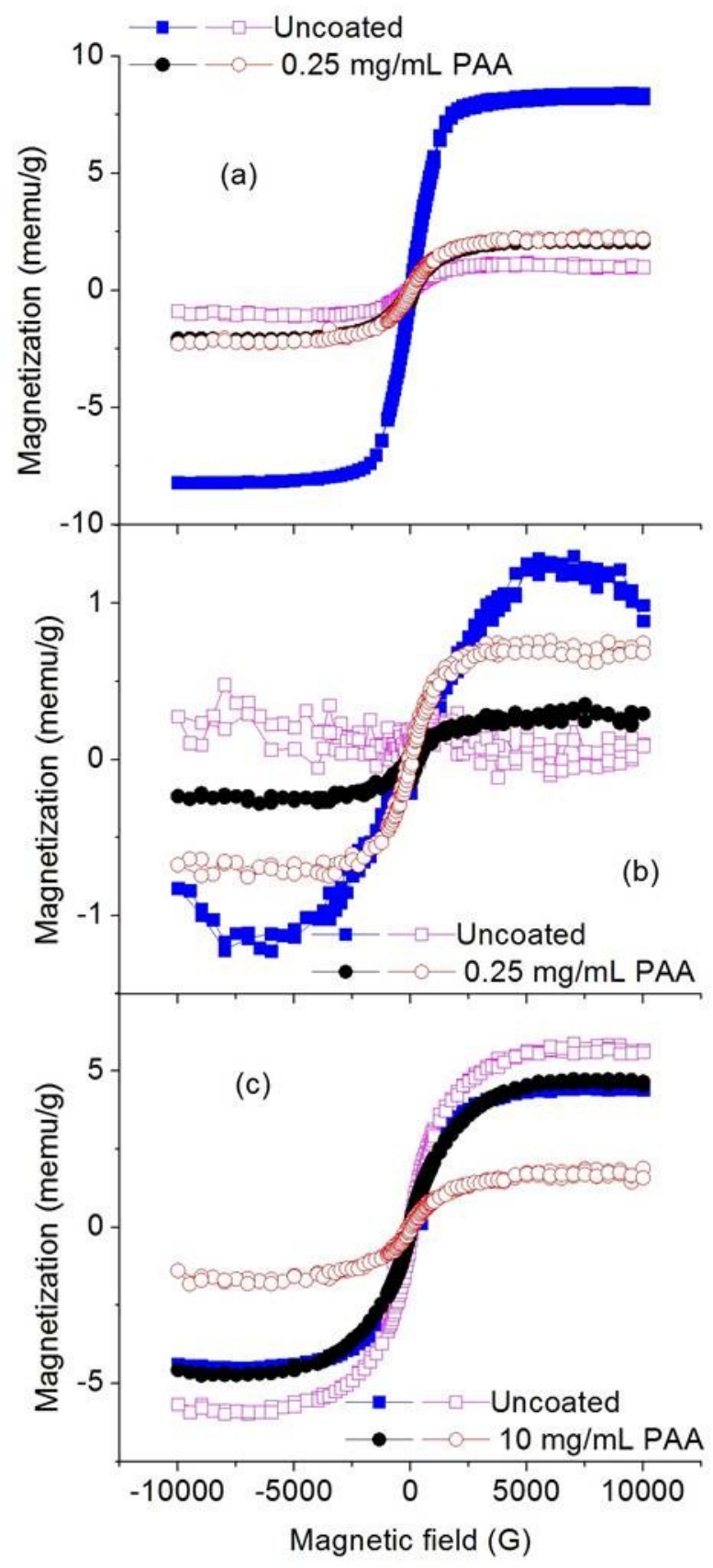

